\title{
Glutamine Requirement for Aerial Mycelium Growth in Neurospora crassa
}

\author{
By MARÍA E. CÁRDENAS AND WILHELM HANSBERG* \\ Centro de Investigación sobre Fijación de Nitrógeno, Universidad Nacional Autónoma de México, \\ Apartado Postal 565-A-Cuernavaca, Mor., Mexico
}

(Received 8 December 1983)

\begin{abstract}
Five amino acids are accumulated during vegetative growth of Neurospora crassa, particularly during the prestationary growth phase. Alanine, glutamine, glutamate, arginine and ornithine comprised over $80 \%$ of the total amino acid pool in the mycelium. Amino acid pools of different amino acid auxotrophs were followed during the partial transformation of a mycelial mat into an aerial mycelium. The mycelial mat under starvation and in direct contact with air rapidly formed aerial mycelium, which produced thereafter a burst of conidia. During this process, glutamine and alanine in the mycelial mat were consumed more rapidly than other amino acids; in the growing aerial mycelium, glutamate and glutamine were particularly accumulated. Of the amino acids that were initially accumulated in the mycelial mat, only a high glutamine pool was required for aerial mycelium growth induced by starvation. This requirement for glutamine could not be satisfied by a mixture of the amino compounds that are synthesized via glutamine amidotransferase reactions. It is proposed that glutamine serves as a nitrogen carrier from the mycelial mat to the growing aerial mycelium.
\end{abstract}

\section{INTRODUCTION}

Mycelium of Neurospora crassa under nitrogen- or carbon-limited growth conditions grows aerial mycelium and conidiates (Turian \& Bianchi, 1972; I. Toledo \& W. Hansberg, unpublished results). Although conidiation of Neurospora under laboratory conditions has been a routine practice for over forty years, little is known about the physiological processes associated with the growth of aerial mycelium and conidiation. Aerial mycelium growth and conidiation in Neurospora offers a convenient model to study cell differentiation. When a mycelial mat is incubated without nutrients and in direct contact with the air, it rapidly grows aerial mycelium. Under these conditions it conidiates in a synchronous fashion (Stine \& Clark, 1967) and the different fungal structures can be isolated in homogeneous preparations in sufficient quantities to allow biochemical analysis.

During aerial mycelium growth induced by starvation, the organism must supply nutrients from the mycelial substratum for growth of the aerial mycelium. Therefore some metabolites should be accumulated or synthesized preferentially in the mycelial mat and utilized in the growing aerial mycelium. Our main object is to detect those physiological and metabolic changes that may shed light on how the aerial mycelium is nourished by the mycelium.

Glutamine plays a central role in nitrogen assimilation and distribution. Nitrogen is assimilated via either the glutamate dehydrogenase-glutamine synthetase pathway or the glutamine synthetase-glutamate synthase pathway and is distributed mainly by the action of amidotransferases and transaminases. Glutamine transamidation is essential for many biosynthetic pathways. Through the activity of the glutamine transminase, the amino nitrogen of glutamine can be distributed to other 2-oxo acids. Besides its role in nitrogen metabolism, glutamine can also serve as a source of carbon and energy, and many eukaryotic cells utilize

Abbreviation: MS, methionine sulphoximine. 
glutamine as an energy source in preference to carbohydrates (Reitzer et al., 1979). Amino acid metabolism during aerial mycelium growth and conidiation has not been explored in Neurospora. Here we present evidence that a high pool of glutamine in the mycelium is required for aerial mycelium growth. In the accompanying paper (Cárdenas \& Hansberg, 1984) we present a detailed description of the enzyme activities related to glutamine metabolism during aerial mycelium growth and propose the pathway through which glutamine serves as a nitrogen source.

\section{METHODS}

Organisms and chemicals. Stock cultures were kindly provided by J. Mora of this University. The strains used were: wild-type strain $74 \mathrm{~A}$, the proline auxotroph prol-3, which grows in proline, ornithine or arginine (Vogel \& Bonner, 1954); the glutamine auxotroph, $g l n-1 b$, which has a defective glutamine synthetase (Dávila $e t$ al., 1983); the tryptophan auxotroph, tryp-2, which grows in indole or tryptophan; and the glutamate auxotroph am-1; en(am)-2, which lacks glutamate dehydrogenase and has no glutamate synthase activity (Hummelt \& Mora, 1980). All reagents were purchased from Sigma.

Growth conditions. Strains were routinely grown on slants with minimal medium N of Vogel (1964), supplemented with $1.5 \%(\mathrm{w} / \mathrm{v})$ sucrose (MM-sucrose) and the required amino acid or amino acid precursor, for $3 \mathrm{~d}$ in the dark at $29^{\circ} \mathrm{C}$, followed by $2 \mathrm{~d}$ in the light at $25^{\circ} \mathrm{C}$. For the gln- $1 b$ strain, MM-sucrose without $\mathrm{NH}_{4} \mathrm{NO}_{3}$ was used, supplemented with $10 \mathrm{mM}$-glutamine. The proline auxotroph was supplemented with $200 \mu \mathrm{g}$ proline $\mathrm{ml}^{-1}$, the tryp-2 strain with $50 \mu \mathrm{g}$ indole $\mathrm{ml}^{-1}$, and the glutamate auxotroph with $5 \mathrm{mM}$-glutamate and $200 \mu \mathrm{g}$ alanine $\mathrm{ml}^{-1}$. Conidia from various slants were resuspended in distilled water and filtered through glass wool. With the conidial suspension, MM-sucrose media, supplemented with the required amino acid or amino acid precursor, were inoculated to give a final $\mathrm{OD}_{540}$ of $0.05 \mathrm{ml}^{-1}\left(10^{6}\right.$ conidia ml-1). MM-sucrose without $\mathrm{NH}_{4} \mathrm{NO}_{3}$, supplemented with $5 \mathrm{mM}$-glutamine, was used for the $g \ln -1 b$ strain. The proline auxotroph was supplemented with $100 \mu \mathrm{g}$ proline $\mathrm{ml}^{-1}$, the tryp-2 strain with $50 \mu \mathrm{g}$ indole $\mathrm{ml}^{-1}$, and the glutamate auxotroph with $5 \mathrm{~mm}$-glutamine and $200 \mu \mathrm{g}$ alanine $\mathrm{ml}^{-1}$. Cultures were incubated for $11-15 \mathrm{~h}$ at $25^{\circ} \mathrm{C}$ with continuous bubbling of humidified air. In experiments to show the requirement of a high glutamine pool, the gln-lb strain was grown for $11 \mathrm{~h}$, then filtered and transferred to MM-sucrose containing $0.1 \mathrm{mM}$-L-methionine sulphoximine (MS) instead of glutamine. After $30 \mathrm{~min}$ incubation in this medium, the culture was divided into equal fractions, filtered separately and resuspended in MM-sucrose without $\mathrm{NH}_{4} \mathrm{NO}_{3}$, in MM-sucrose, or in MM-sucrose supplemented with either $5 \mathrm{~mm}$-glutamine, $10 \mathrm{mM}$-glutamate, $2.5 \mathrm{~mm}$-arginine, $5 \mathrm{~mm}$-tryptophan, $2 \mathrm{mM}$-guanosine or $10 \mathrm{~mm}$-glucosamine, or a mixture of the amino compounds, except glutamine, plus $3.3 \mathrm{~mm}$-histidine, $5 \mathrm{~mm}$-asparagine and $2 \mathrm{~mm}$ adenosine. After another $30 \mathrm{~min}$ incubation, the mycelia were starved to induce growth of aerial mycelium. In other experiments, the amino acid auxotrophs were grown for $12 \mathrm{~h}$ as described, then transferred to a medium without the required amino acid, and incubated for another $2 \mathrm{~h}$ before they were put to grow aerial mycelium.

Growth of aerial mycelium. As a source of mycelia, $300 \mathrm{ml}$ liquid culture was filtered through Whatman no. 41 filter paper. The mycelial mats were washed with $100 \mathrm{ml}$ cold water and $100 \mathrm{ml}$ on $0.03 \mathrm{M}$-phosphate buffer, pH 6.1 (Stine \& Clark, 1967). After eliminating excess buffer, the mycelial mats were transferred into Petri dishes and incubated in a chamber at $80 \%$ relative humidity and $28{ }^{\circ} \mathrm{C}$. After different incubation times, a Petri dish was taken out of the chamber and the aerial mycelium was separated from the mycelial mat with a spatula. Then the mycelial mat was washed with a jet of water in order to separate the loose mycelium from the very tightly adhering mycelium of the uppermost layer (about $10 \%$ of the mycelial mat). All three mycelia were collected in cold water, filtered through Whatman no. 41 filter paper, and dehydrated with acetone. Mycelia were used directly, or vacuum dried and stored at $-20^{\circ} \mathrm{C}$.

Amino acid determination. The mycelia were boiled for $10 \mathrm{~min}$ in $80 \%(\mathrm{v} / \mathrm{v})$ ethanol solution, homogenized and filtered through Millipore RA $1.2 \mu \mathrm{m}$ filters. The filtrates were lyophilized and stored at $-20^{\circ} \mathrm{C}$. Samples were resuspended in deionized water $(1 \mathrm{ml}$ per $\mathrm{mg}$ mycelial protein), and protein was determined by the Lowry method. The amino acids were separated on an Aminco amino acid analyser and quantified using an Aminco ratio fluorimeter (Aminco, Silver Spring, Md, USA) after coupling with $o$-phthaldialdehyde.

\section{RESULTS}

\section{Aerial mycelium growth under starvation conditions}

Using the method described, aerial mycelium was seen over the entire surface of the mycelial mat after $2 \mathrm{~h}$ incubation. It then grew rapidly for the next $10-12 \mathrm{~h}$ and conidiated thereafter in a burst of $2 \mathrm{~h}$. After a $15 \mathrm{~h}$ incubation period, there was hardly any further increment in aerial hyphae biomass, measured as total protein content. The top layer of the mycelial mat, in direct 


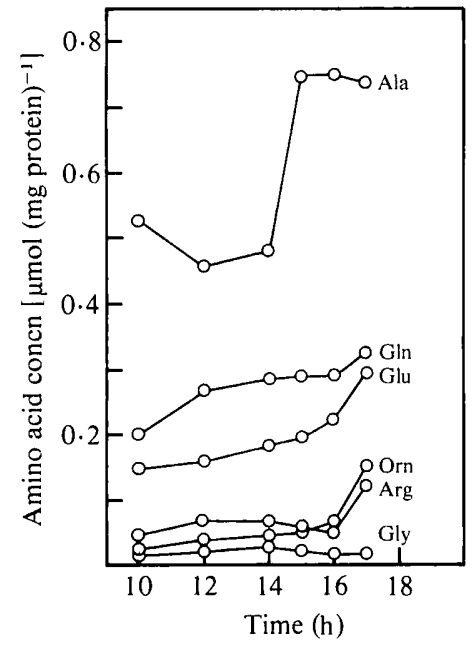

Fig. 1. Accumulation of amino acids in the mycelium during the prestationary growth phase. Other amino acid pools were similar to Gly (Asp, Thr, Ser, Val) or lower; His and Lys pool levels could not be accurately determined.

contact with the air, acquired the consistency of a tight tissue, which could therefore be easily separated from the lower layers of the mycelial mat and analysed separately.

The conidia obtained from starved mycelium were viable: they formed a high percentage of colonies (93\% on the sixth day) and when inoculated into liquid media they developed a mycelium which grew with a biomass doubling time of $2.5 \mathrm{~h}$. This mycelium was again able to conidiate with a similar efficiency to mycelia grown from conidia that were harvested from slants.

\section{Amino acid pools during aerial mycelium growth}

In order to detect changes in amino acid metabolism, particularly that of glutamine, amino acid pools were determined before and during the whole process of aerial mycelium growth. Amino acids were accumulated in the mycelium of the prestationary growth phase, they decreased during the formation of the aerial hyphae, and were again accumulated in the aerial mycelium. During the prestationary growth phase, alanine, glutamine, glutamate, ornithine and arginine were accumulated in the mycelium (Fig. 1). Similar values for glutamine and arginine have been reported by Mora et al. (1978). At $15 \mathrm{~h}$ growth, alanine alone accounted for $51 \%$ of the total amino acid pool; glutamine, 19.6\%; glutamate, $13.3 \%$; ornithine, $4 \%$; and arginine, $4 \%$ (Fig. 2a).

When the mycelium of the prestationary growth phase $(15 \mathrm{~h})$ was starved to induce growth of aerial mycelium, the amino acid pools decreased. By the end of the aerial mycelium growth $(15 \mathrm{~h})$ all amino acid pools in the mycelial mat had decreased, with the possible exception of glycine (Fig. $2 a, b$ ). There was a drastic fall in the alanine and glutamine pools. The rate of consumption in the mycelial mat during aerial mycelium growth was similar for most of the amino acids; only glutamine and alanine seemed to decrease at a higher rate, particularly between 3 and 6 h (Fig. 3).

In the conidiating aerial mycelium there was a particularly high pool of glutamate $(34 \%$ of the total amino acid pool). Other amino acids that were accumulated in the aerial mycelium were: glutamine, $19 \%$; alanine, $17.5 \%$; and ornithine, $6.5 \%$. Serine, aspartate, and threonine pools were higher in the aerial mycelium than in the mycelium of the prestationary growth phase (Fig. $2 a, c)$. 


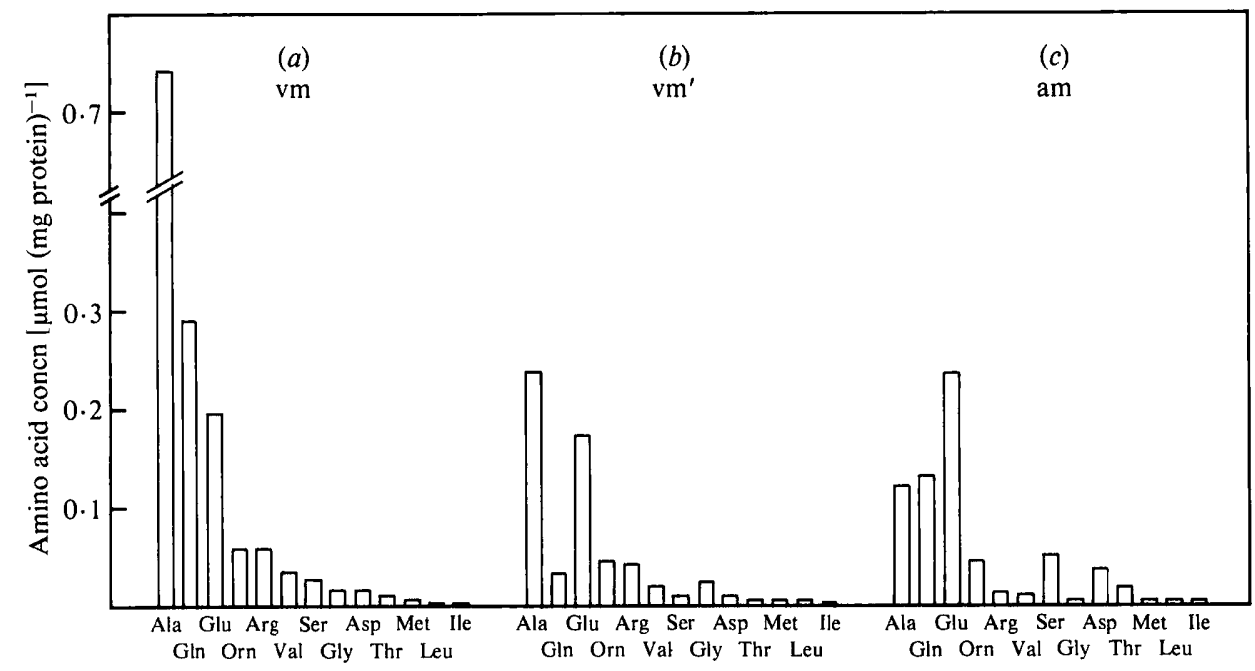

Fig. 2. Amino acid pools in (a) the mycelium at the prestationary growth phase (vm, $15 \mathrm{~h}),(b)$ the mycelial mat after $15 \mathrm{~h}$ aerial mycelium growth $\left(\mathrm{Vm}^{\prime}\right)$ and $(c)$, the aerial mycelium at $15 \mathrm{~h}$ growth (am). Pools of amino acids that were less than $5 \mathrm{nmol}$ (mg protein) $)^{-1}$ are not shown. His and Lys pools could not be accurately determined.

\section{Requirement for a high mycelium glutamine pool for aerial mycelium growth}

Mycelium of $N$. crassa accumulates glutamine during prestationary growth and under conditions in which growth is restricted (Mora et al., 1978). Accumulation of glutamine during exponential and prestationary growth could be required for the growth of aerial mycelium. Therefore, the capacity of different amino acid auxotrophs to form aerial hyphae was tested. Glutamine $(g \ln -1 b)$ and glutamate $(a m-1 ; e n(a m)-2)$, and three other amino acid auxotrophs, arginine (arg-3), tryptophan (tryp-2), and proline (prol-3) were starved to induce growth of aerial mycelium. They all formed aerial hyphae, although with different efficiency (Fig. 4). The same auxotrophs were incubated for $2 \mathrm{~h}$ in a medium without amino acid, in order to deplete the pool of the required amino acid, before putting them to grow aerial mycelium. Under these conditions, all strains grew aerial hyphae; only $g l n-1 b$ showed a marked reduction in the amount of aerial mycelium formed: $72.5 \%$ less than the non-deprived control. The arg-3 strain grew $26.6 \%$ less, and prol-3, am- 1 ; en(am)-2, and tryp-2 mutants formed, respectively, 9,15 and $8 \%$ more aerial mycelium than their non-depleted controls (Fig. 4). In each case, the amino acid pools were measured in the mycelia before they were starved to induce growth of aerial mycelium (Table 1). Proline was not detected because of the method used for amino acid determination, but its pool is probably non-detectable (Schmit \& Brody, 1975). The tryptophan pool was also non-detectable in the strains used. It could only be determined in tryp- 2 grown in the presence of indole. In this strain, the tryptophan pool disappeared after incubation for $2 \mathrm{~h}$ in a medium without indole. After amino acid starvation, the amino acid pool levels were as follows. ( $a$ ) In the gln-1b mutant the glutamine pool was 7-14-fold lower than in the other strains; consequently, the arginine and histidine pools were lower, and the glutamate and ornithine pools higher, than in the other strains (ornithine and histidine are not shown in Table 1). (b) In the am-1; en(am)-2 double mutant, the glutamate pool was 3-6-fold lower, while the glutamine pool was only 2-fold lower than in the other strains. (c) In the arg-3 mutant the arginine pool was 2-3fold lower than in the other strains; a complete depletion of arginine is not possible without depleting the glutamate pool (Drainas \& Weiss, 1982).

Under conditions in which glutamine was utilized as a nitrogen source, a marked decrease in the alanine pool was observed: the $g l n$ - $1 b$ mutant, grown with glutamine, had a 1.5-2-fold lower pool of alanine than the other strains; the $a m-1$; en(am)-2 double mutant had an even lower alanine pool after $4 \mathrm{~h}$ in a medium with alanine plus glutamine: $3-4$-fold lower than in other 


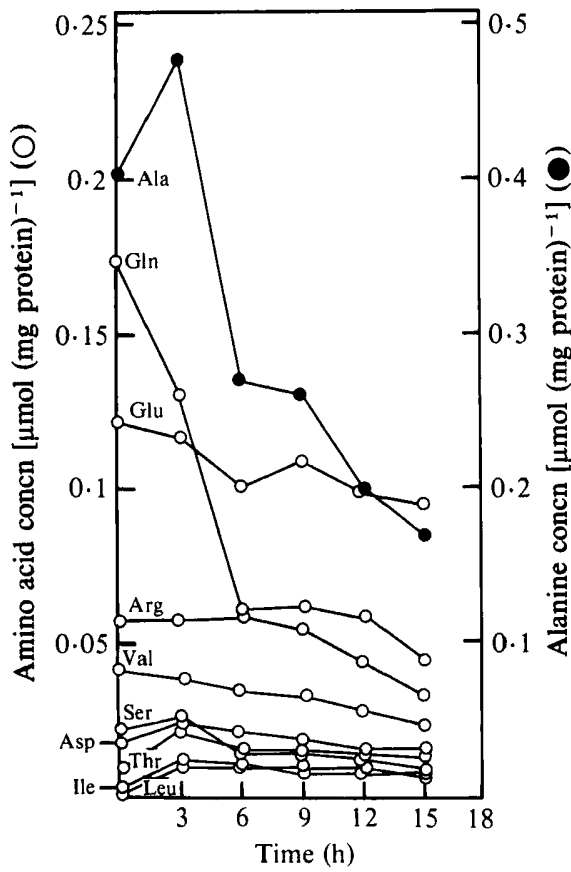

Fig. 3

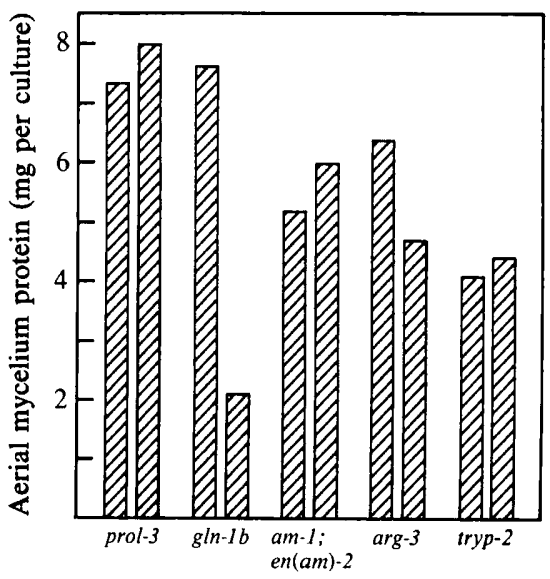

Fig. 4

Fig. 3. Amino acid pools in the mycelial mat during the course of aerial mycelium growth. Pools lower than $5 \mathrm{nmol}$ (mg protein) $)^{-1}$ are not shown. His and Lys pools could not be accurately determined.

Fig. 4. Aerial mycelium growth of different amino acid auxotrophs. The required amino acid pool was either not depleted (first column for each strain) or depleted (second column) just before the auxotrophs were induced to grow aerial mycelium.

strains (Table 1). When these strains were transferred thereafter to an ammonium-containing medium, the alanine pool in $g l n-1 b$ increased over 4 -fold after $2 \mathrm{~h}$, while in $a m-1 ;$ en $(a m)-2$ it decreased 7-fold (Table 1).

\section{Restoration of mycelium growth with restoration of the glutamine pool}

Since the $g \ln -1 b$ mutant was markedly affected in its ability to form aerial mycelium when depleted of glutamine, different compounds besides glutamine were tested for their capacity to restore aerial growth. The $g l n-1 b$ strain was grown for $12.5 \mathrm{~h}$ in a medium containing glutamine, then transferred to a medium without glutamine but with $0.1 \mathrm{~mm}-\mathrm{MS}$, incubated for $30 \mathrm{~min}$, and thereafter transferred to a medium with either glutamine, ammonium nitrate, or other organic nitrogen compounds. MS, a highly specific inhibitor of glutamine synthetase (Ronzio \& Meister, 1968), was used in order to inhibit the glutamine synthetase $\alpha$ of the $g l n-1 b$ strain (Dávila et al., 1983). Glutamine synthetase specific activity after $30 \mathrm{~min}$ incubation with the inhibitor was $0.0004 \mathrm{U}$ (mg protein $)^{-1}, 7$-fold lower than without the inhibitor, and 175 -fold lower than the $74 \mathrm{~A}$ wild-type strain grown with glutamine as the nitrogen source (Dávila et al., 1978). When the gln$1 b$ strain was treated with MS and thereafter incubated for $30 \mathrm{~min}$ in a medium with glutamine in order to restore the glutamine pool just before starvation, abundant growth of aerial mycelium was obtained (Fig. 5). If, instead of restoring the glutamine pool of the strain, it was incubated in a medium containing either ammonium nitrate, glutamate, arginine, tryptophan, guanosine or glucosamine, the aerial mycelium growth was $38-71 \%$ lower than that obtained when the strain was incubated with glutamine (Fig. 5). A mixture of the former organic nitrogen compounds plus histidine, asparagine and adenosine was unable to restore normal aerial mycelium growth. 


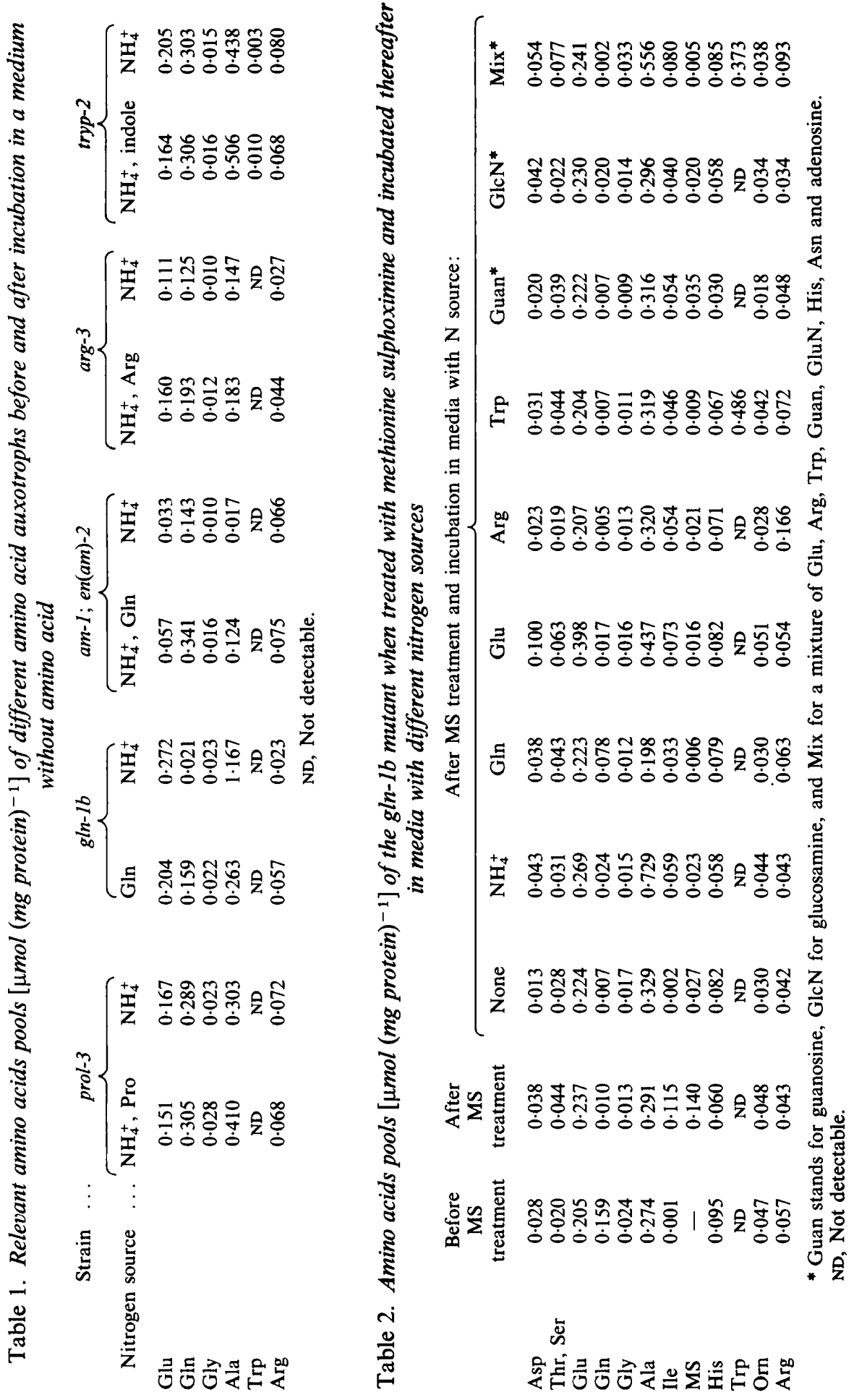




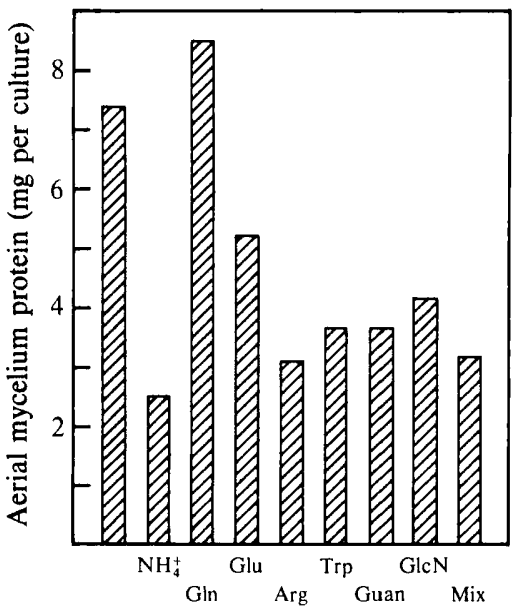

Fig. 5. Aerial mycelium growth of the $g l n-l b$ strain when incubated in different media before induction of aerial mycelium growth. First column, glutamine pool in the mycelium not depleted; remaining columns, glutamine pool depleted by incubation in a medium without glutamine but with $0.1 \mathrm{mM}-\mathrm{L}$ methionine sulphoximine and followed by transfer to a medium with either $\mathrm{NH}_{4} \mathrm{NO}_{3}$, Gln, Glu, Arg, Trp, guanosine, glucosamine $(\mathrm{GlcN})$ or a mixture (Mix) of all amines except Gln, plus Asn, His and adenosine.

The pools of glutamate, arginine, tryptophan, histidine and glucosamine increased when these compounds were present in the incubating medium (Table 2). Guanosine, adenosine and asparagine pools were not measured.

Besides its effect on glutamine depletion and the consequent decrease in the histidine and arginine pools, MS also increased the pools of glutamate, threonine plus serine, alanine, aspartate and, most importantly, isoleucine (Table 2). When the culture treated with MS was then incubated without a nitrogen source, the high isoleucine pool disappeared, the MS peak in the amino acid profile decreased strongly (Table 2), but a high peak corresponding to the change from buffer $\mathrm{pH} 2.88$ to $\mathrm{pH} 4.15$ was observed. These results are consistent with MS being a substrate for $\gamma$-cystathionase (Cooper et al., 1976).

\section{Amino acid pools in different amino acid auxotrophs during aerial mycelium growth}

The amino acid pools of the prol-3, gln-1b, am-1; en(am)-2, arg-3 and tryp-2 strains were measured at the end of aerial mycelium growth in the top layer of the mycelial mat, in the rest of the mycelium (lower layers), and in the aerial mycelium. This was done both when the strains were depleted of their required amino acid before aerial mycelium induction, and without depletion. The prol-3 and tryp-2 mutants grew the normal amount of aerial mycelium whether depleted or not (Fig. 4), and had very similar amino acid pools under both conditions. In both strains, similar amino acid pools in the different fungal structures were observed. The arg-3 strain also had similar amino acid pools, but lower pools of arginine and glutamine, particularly when the strain had been incubated without arginine. Under this condition arg-3 formed $26 \%$ less aerial mycelium than when it had normal pools of arginine and glutamine. Since the amino acid pools were similar in all three strains, with the exceptions mentioned, only the relevant amino acid pools of the prol-3 strain are shown in Fig. 6. It can be seen that the glutamine pool in the mycelial mat was almost depleted and that a gradient of glutamine had been formed between the lower layers, the top layer of the mycelial mat, and the aerial mycelium. A similar situation was observed in the $g \ln -1 b$ strain when the glutamine pool was not depleted before starvation to form aerial mycelium. Only higher pools of glutamate and lower pools of glutamine were observed in the lower layers of the mycelial mat and in the aerial mycelium (Fig. $6 c$ ). However, when the glutamine pool of the $g l n-l b$ mutant was depleted before induction of aerial mycelium growth, aerial mycelium grew sparsely (Fig. 4) and had very low pools of all amino acids, even 


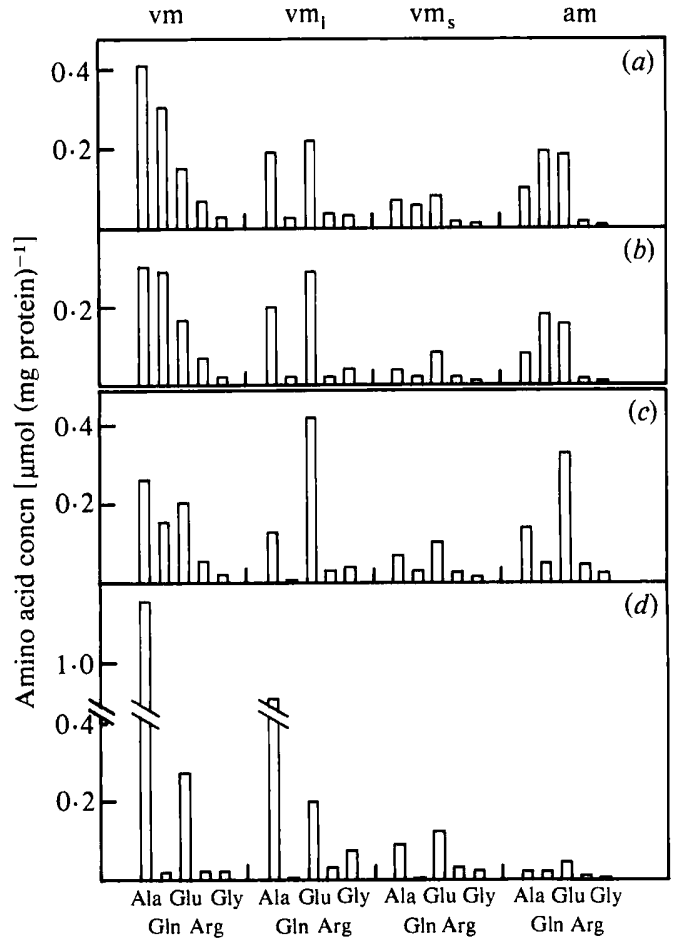

Fig. 6

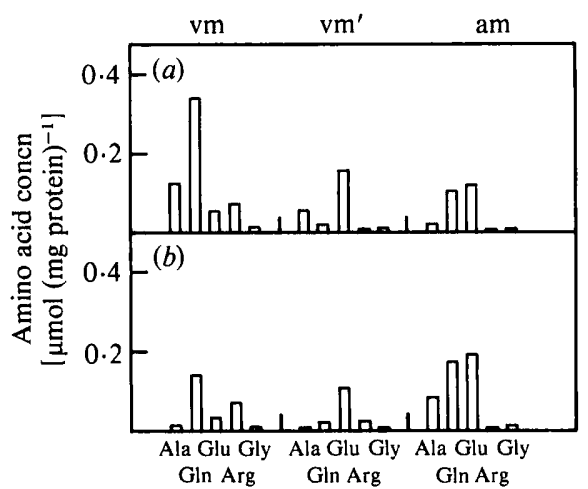

Fig. 7

Fig. 6. Pools of the accumulated amino acids in the different fungal structures of the prol-3 and $g \ln -1 b$ strains in the mycelium at the end of the exponential growth phase (vm); in the lower layers $\left(v m_{i}\right)$ and the top layer $\left(\mathrm{vm}_{\mathrm{s}}\right)$ of the mycelial mat at the end of aerial mycelium growth; and in the aerial mycelium $(\mathrm{am})$ at the end of its growth. (a) prol-3 untreated; $(b)$ prol-3 incubated for $2 \mathrm{~h}$ in a medium without proline; (c) $g \ln -1 b$ untreated; $(d)$ gln- $l b$ incubated for $2 \mathrm{~h}$ in a medium without glutamine.

Fig. 7. Pools of the accumulated amino acids in the different fungal structures of the am- 1 ; en $(\mathrm{am})-2$ strain, in the mycelium at the end of the exponential growth phase (vm); in the mycelial mat, top and lower layers together, at the end of aerial mycelium growth $\left(\mathrm{vm}^{\prime}\right)$; and in the aerial mycelium at the end of its growth (am). (a) Untreated; $(b)$ incubated for $2 \mathrm{~h}$ in a medium without amino acid.

though there were high pools of amino acids in the mycelial mat (Fig. 6d). The am-1; en(am)-2 strain grew a normal amount of aerial mycelium, whether the glutamate and alanine pools were depleted or not, and under both conditions the amino acid pools in the aerial mycelium were similar to prol-3 (Fig. $7 a, b$ ). It is particularly noteworthy that the am-1; en(am)-2 strain, although lacking biosynthetic glutamate dehydrogenase and glutamate synthase activities, could accumulate glutamate in the aerial mycelium.

\section{DISCUSSION}

When $N$. crassa is grown in solid media with an abundance of nutrients, it grows aerial mycelium from the second to the fifth day of incubation. During this period, part of the culture grows vegetatively while other parts are already engaged in aerial mycelium growth or conidiation. By growing the culture in a liquid medium and then exposing it to the air in the absence of nutrients, the vegetative growth period is separated from the fruiting period. This is a requisite for comparison of the metabolism of the mycelium when it is growing or forming aerial hyphae.

Under starvation conditions, the aerial mycelium grew rapidly and evenly over the entire airexposed surface of the mycelial mat, and thereafter synchronous conidiation took place. At any 
time after $5 \mathrm{~h}$ incubation, aerial mycelium could be harvested and the mycelial mat separated into the top layer, the layer exposed to the air, and the mycelium below. All three structures are morphologically and biochemically distinct (Cárdenas \& Hansberg, 1984). It is important to stress that study of aerial mycelium growth and conidiation requires separate analysis, of the different structures, and to our knowledge this is the first time that this has been attempted.

During exponential growth, the levels of alanine, glutamine, glutamate, ornithine and arginine were very high: together they accounted for over $80 \%$ of the total amino acid pool (Fig. 1). Very similar values have been reported by Schmit \& Brody (1975). The alanine pool was by far the largest, amounting to $50 \%$ of the total. During the prestationary growth phase, these five amino acid pools increased even further. Alanine accumulated to a high concentration 1 or $2 \mathrm{~h}$ before the glutamate, glutamine, ornithine and arginine pools started to increase (Fig. 1). In liquid media, prestationary growth was due to exhaustion of carbon and nitrogen sources, since the addition of carbon and/or nitrogen sources prolonged the exponential growth by $3-4 \mathrm{~h}$, and increased the yield at the stationary phase (I. Toledo \& W. Hansberg, unpublished results). It is remarkable that when carbon and nitrogen sources were limited, the pools of the five amino acids mentioned increased even further. Under other non-growing conditions, glutamine and arginine are also accumulated (Espín \& Mora, 1978; Espín et al., 1979). That the alanine pool increased before those of glutamate, glutamine, ornithine and arginine could be explained by the fact that pyruvate strongly inhibits glutamine synthetase activity (M. E. Cárdenas \& W. Hansberg, unpublished results). Thus when growth rate was declining but pyruvate was still available, glutamine synthetase activity could be partially inhibited by pyruvate, and glutamate drained towards alanine formation by transamination with pyruvate; when pyruvate became more limiting, alanine would no longer be synthesized, glutamate would accumulate, and more glutamine would be synthesized, since glutamine synthetase would no longer be inhibited by pyruvate. As another consequence, the pool levels of ornithine and arginine would increase.

A relation between the alanine and the glutamine pools was also detected when these pools were measured in the different amino acid auxotrophs used. When glutamine was used as a nitrogen source, a marked decrease in the alanine pool was observed (Tables 1 and 2). Since no glutaminase activity could be detected in $N$. crassa (Cárdenas \& Hansberg, 1984), glutamine may be catabolized via the glutamine transminase- $\omega$-amidase pathway (Cooper \& Meister, 1977). This pathway has been found to be active in N. crassa (J. Calderón, E. Morett \& J. Mora, unpublished). The 2-oxoglutarate resulting from $\omega$-amidase activity would drive the alanine transaminase activity towards the formation of glutamate and pyruvate, and could consequently lower the pool of alanine. This could be particularly the case in the $a m-1$; en $(a m)-2$ strain, which lacks the NADP-glutamate dehydrogenase and the glutamate synthase activities needed for glutamate synthesis. This would explain why, when this strain is transferred to a medium without glutamate, its pool of glutamine continues to be drained and why the alanine pool is greatly depleted. By contrast, the $g l n-1 b$ strain, which has normal levels of NADP-glutamate dehydrogenase, actively synthesizes glutamate and hence accumulates alanine by transamination when transferred to an ammonium medium.

Since aerial mycelium growth in Neurospora is triggered by limitation in the carbon and nitrogen sources (Turian \& Bianchi, 1972; I. Toledo \& W. Hansberg, unpublished results), the accumulated amino acids in the mycelium could serve as internal carbon and nitrogen reservoirs to be used during the growth of aerial mycelium and conidiation. Pools of all amino acids in the mycelial mat, with the possible exception of glycine, were lower at the end of the aerial mycelium growth than during the prestationary growth (Fig. $2 a, b$ ), but the alanine and glutamine pools were consumed much faster than the other amino acid pools, predominantly during the first $6 \mathrm{~h}$ of aerial mycelium growth (Fig. 3).

Of all the accumulated amino acids in the vegetative mycelium, only glutamine seemed to be indispensable for aerial mycelium growth. Very low pools of glutamate and alanine were found in the $a m-1 ; e n(a m)-2$ double mutant when incubated in the absence of amino acid, and relatively low pools of arginine and ornithine were found in the arg-3 mutant when incubated in the absence of arginine (Table 1). Under these conditions, both strains retained their capacity to form aerial mycelium. However, the $g l n-l b$ mutant formed very little aerial mycelium when 
incubated without glutamine (Fig. 4). Only when the glutamine pool was restored to its normal level did the $g l n-1 b$ mutant regain its capacity to form aerial mycelium (Fig. 5). These experiments suggest that a high glutamine pool in the mycelial mat is actually required for aerial mycelium growth. This requirement could not be satisfied by a mixture of all the amino compounds that are synthesized via the glutamine amidotransferase reactions. It seems probable that the high glutamine requirement is related to the nitrogen supply for the growing aerial mycelium. A hypothesis consistent with the results is that, of the major pool amino acids, only glutamine can be efficiently exported from the mycelial mat to the developing aerial hyphae.

We are grateful to Jaime Mora for this criticism throughout this work. We also thank Ivonne Toledo for her technical assistance, Emelia Breña-Valle for critically reading the manuscript and Guadalupe Dingler for her secretarial assistance.

This research was supported in part by CONACyT (Consejo Nacional de Ciencia y Tecnología) México, grant PCCBNAL790056 to W. Hansberg, María E. Cárdenas was supported by an UNAM-CONACyT fellowship.

\section{REFERENCES}

Cárdenas, M. E. \& Hansberg, W. (1984). Glutamine metabolism during aerial mycelium growth of Neurospora crassa. Journal of General Microbiology 130, 1733-1741.

Cooper, A. J. L., Stephani, R. A. \& Meister, A. (1976). Enzymatic reactions of methionine sulfoximine. Journal of Biological Chemistry 251, 66746682.

CoOper, A. J. L. \& Meister, A. (1977). The glutamine transaminase- $\omega$-amidase pathway. CRC Critical Reviews in Biochemistry 4, 281-303.

Dávila, G., Sánchez, F., Palacios, R. \& Mora, J. (1978). Genetics and physiology of Neurospora crassa glutamine auxotrophs. Journal of Bacteriology 134, 693-698.

Dávila, G., Brom, S., Mora, Y., Palacios, R. \& MORA, J. (1983). Genetic and biochemical characterization of glutamine synthetase from Neurospora crassa glutamine auxotrophs and their revertants. Journal of Bacteriology 156, 993-1000.

Drainas, C. \& WeIss, R. L. (1982). Energy requirement for the mobilization of vacuolar arginine in Neurospora crassa. Journal of Bacteriology 150, 779784.

Espí, G., \& MORA, J. (1978). Effect of the deprivation of amino acids on conidia of Neurospora crassa. Journal of General Microbiology 104, 233-240.

Espín, G., Palacios, R. \& Mora, J. (1979). Glutamine metabolism in conidia from Neurospora crassa. Journal of General Microbiology 115, 59-68.

Hummelt, G. \& Mora, J. (1980). Regulation and function of glutamate synthase in Neurospora crassa. Biochemical and Biophysical Research Cummunications 96, 1688-1694.

MorA, Y., Espín, G., Willms, K. \& Mora, J. (1978). Nitrogen accumulation in mycelium of Neurospora crassa. Journal of General Microbiology 104, 241-250.

Reitzer, L. J., WiCe, B. M. \& KenNell, D. (1979). Evidence that glutamine, not sugar, is the major energy source for cultured HeLa Cells. Journal of Biological Chemistry 254, 2669-2676.

Ronzio, R. A. \& Meister, A. (1968). Phosphorylation of methionine sulfoximine by glutamine synthetase. Proceedings of the National Academy of Sciences of the United States of America 59, 164-170.

SCHMIT, J. C. \& BRoDY, S. (1975). Neurospora crassa conidial germination: role of endogenous amino acid pool. Journal of Bacteriology 124, 232-242.

Stine, G. J. \& ClaRK, A. M. (1967). Synchronous production of conidiophores and conidia of Neurospora crassa. Canadian Journal of Microbiology 13, 447-453.

Turian, G. \& Bianchi, D. E. (1972). Conidiation in Neurospora. Botanical Review 38, 119-154.

Vogel, H. J. \& Bonner, D. M. (1954). On the glutamate-proline-ornithine interrelations in Neurospora crassa. Proceedings of the National Academy of Sciences of the United States of America 40, 688694.

VoGeL, H. J. (1964). Distribution of lysine pathways among fungi: evolutionary implications. American Naturalist 98, 435-446. 